\title{
Nonlinear dynamics, identification and monitoring of structures: a special issue dedicated to the memory of Francesco Benedettini
}

\author{
Angelo Luongo • Giuseppe Rega
}

Published online: 18 October 2016

(C) Springer Science+Business Media Dordrecht 2016

\section{Preface}

Francesco Benedettini, Professor of Strength of Materials and of Dynamics of Structures at the University of L'Aquila, passed away on 15 February 2015 at the age of 59, in his full maturity as a men, a professor and a scholar. It was a very premature and painful loss not only for his family but also for the communities of national scientists in solid and structural mechanics, and of international scholars of nonlinear and structural dynamics.

Upon graduating at the University of Padua in 1979, Francesco was appointed at the University of L'Aquila as an assistant professor in 1984, as an associate professor in 1992, and as a full professor in 2000. Therein, he was responsible of the Experimental Laboratory of Nonlinear Dynamics (later on expanded to include meaningful In-situ Dynamic Testing) since its establishment in 1992. He was also chair of Master (Civil and Infrastructure Engineering; Earthquake Engineering) and Ph.D. (Civil and Environmental Engineering) programs.

\footnotetext{
A. Luongo $(\square)$

Department of Civil, Construction-Architectural and Environmental Engineering, University of L'Aquila, L'Aquila, Italy

e-mail: angelo.luongo@ing.univaq.it

G. Rega

Department of Structural and Geotechnical Engineering,

Sapienza University of Rome, Rome, Italy
}

Francesco contributed meaningful and well recognized research on nonlinear vibrations of systems and structures for nearly 30 years, masterly using the combination of advanced techniques-analytical, computational, geometrical, and experimentalneeded to carefully detect and reliably characterize a variety of nonlinear and complex dynamic phenomena possibly occurring in different engineering areas.

Within this general framework, Francesco's constant interest, among many others, was devoted to the finite amplitude oscillations of elastic monodimensional systems with initial curvature (suspended cables and arches). At first, upon obtaining suitable reduced order models of the underlying partial differential equations, he developed involved highorder multiple time scale solutions of the ensuing ordinary equations, which allowed to highlight the effects of quadratic and cubic nonlinearities under different external and internal resonance conditions [1-3]. Yet, grasping the increasing importance of bifurcation and chaos phenomena, he was soon among the first to implement advanced numerical and geometrical techniques for the analysis, prediction and diagnosis of bifurcation events in structural dynamics [4, 5], while at the same time starting a meaningful (and indeed earlier) activity of experimental nonlinear dynamics on a variety of flexible mechanical models [6-9] with the aim to crossvalidate theoretically observed phenomena via sophisticated techniques of reconstruction of their physical response [10-12]. 
While remaining always active in this field, in the last 15 years of his activity Francesco shifted his main research interests towards more applicative problems in the area of structural mechanics and engineering, focusing on the identification and monitoring of structures. Indeed, resuming the earlier attention paid to dynamic identification problems [13], he provided updated contributions on experimental modal analysis in operational conditions [14], on damage identification paralleled with model updating [15], and on monitoring the dynamical properties of a great variety of bridges [16, 17] and other important structures subjected to ambient vibrations, including traffic and earthquakes [18]. As a matter of fact, in this broad area he was also the chairman of the International Operational Modal Analysis Conference (IOMAC) since 2013.

It is certainly worth noting that, in addressing such application-oriented problems within a structural health monitoring perspective, Francesco suitably exploited all of his expertise on the advanced dynamic behavior of systems previously gained in the more theoretically-oriented stage of his scientific activity.

Overall, a distinguishing feature of Francesco's scientific research was to constantly complement theoretical and numerical investigations with the advanced use and the innovative implementation of experimental techniques suitable to analyze the response of systems at both the small-scale of a specialized nonlinear dynamics laboratory and the large-scale of real structures in civil engineering.

Francesco was an open-minded, determined, and tireless scholar, full of brilliant initiatives (some of which unfortunately unfinished), as well as of human richness and availability towards students and colleagues. He was indeed a true gentleman in both behavior and, even more, in the depth of his soul.

We aim now at honoring his memory as a man and a scholar, by recognizing his contribution to the advancement of knowledge in nonlinear dynamics, identification, and monitoring of structures through the publication of a Special Issue of Meccanica. As a natural choice, scholars active in these areas, who had scientific and personal involvements with Francesco, were basically invited to contribute.

However, as a witness of his capability and richness of friendly relationships and personal feelings, we were equally happy to invite a number of Francesco's colleagues and friends preferably active in slightly different, though companion, fields.

This has resulted in a Special Issue which collects 22 papers that have been regularly reviewed and are now grouped in three different sections. Thirteen of them are concerned with topics in Nonlinear Dynam$i c s$, ranging from the analysis of nonlinear vibrations of various systems up to different problems of dynamics and stability, dynamics and control, and impulsive dynamics. Herein, a rich variety of models are considered, along with different approaches and scientific perspectives. The following seven papers, grouped under the heading of Identification and Monitoring of Structures, encompass topics in modal analysis, identification, and damage characterization, with the attention mostly paid to the experimental behavior of real structures of also historical interest. The last two papers deal with problems in Solid Mechanics.

In the sequel, we shortly report on the specific content of each paper.

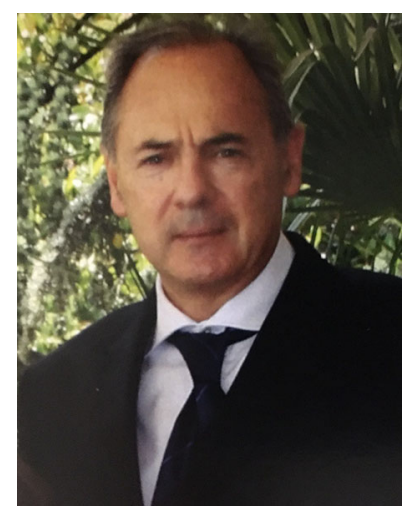

\subsection{Nonlinear dynamics}

Five papers deals with free or forced nonlinear vibrations of different systems, basically addressed via asymptotic methods.

Warminski, Zulli, Rega and Latalski resume a fourdegree-of-freedom model of suspended cable proposed in the most cited of Francesco's papers ([3], with 95 citations, according to Scopus), showing its potential for addressing the multimodal nonlinear response of a multiple internally resonant sagged cable, subjected to a variety of excitation conditions involving supports motion. A cancellation phenomenon entailed in the system response by the 
addition of external loading is highlighted, along with numerically detected regimes of complex dynamics.

Dealing with another system addressed by Francesco in his scientific activity [9], Lakrad, Chtouki and Belhaq investigate the nonlinear vibrations of a twodegree-of-freedom model of a shallow arch subject to a resonant external harmonic forcing and to a very slow harmonic imposed displacement of one of its supports. Zones of various periodic bursters and chaotic dynamics are detected, showing the beneficial effect of the slow parametric excitation, with also small amplitudes, in possibly suppressing chaos from wide regions of control parameters space.

Lenci, Clemente and Rega study the free nonlinear oscillations of planar elastic beams. Exact equations of motion, encompassing shear deformation and rotatory inertia, are considered. They are based on a nonstandard expression for the curvature, which is believed mechanically more consistent. It is shown that geometrical and mechanical parameters, including the stiffness of a spring added at one end, are responsible for qualitative changes in the beam behavior, namely from softening to hardening. Low slenderness beams are also studied.

Vortex-induced vibrations of turbine blades at 1:1 internal resonance are investigated by Wang, Chen, Wiercigroch and Cao via a three-degree-of-freedom model accounting for bending-torsion coupling and fluid-blade interaction. Dynamic response and bifurcation characteristics with respect to detuning and freestream velocity are obtained, and the influence of system parameters (lift and moment coefficients, structural damping, cubic nonlinearity, and other coupling parameters) is analyzed, with a view to possibly optimizing their values to avoid fatigue of blades.

Lacarbonara, Carboni and Quaranta explore the use of nonlinear normal modes (NNMs) to detect damage in simply supported beams affected by a localized reduction of flexural stiffness. The backbones curves and the NNMs of the system are derived by the multiple scale method. Damage is investigated by comparing the curvatures of the backbones curves, which are found to be more sensitive than the linear frequencies. Useful information about the location of damage are also drawn by a proposed effective identification strategy, which is robust against noise.

Issues of dynamics and stability, dynamics and control, or impulsive dynamics are addressed in the following eight papers, via different techniques applied to a variety of systems.

Luongo, D'Annibale and Ferretti discuss the effects of nonlinear hysteretic damping on the post-critical behavior of the classical Ziegler's column, endowed with nonlinear dashpots of Van der Pol-type at the hinges. Supercritical or subcritical Hopf bifurcations and the occurrence of the 'hard loss of stability' phenomenon are investigated via a second-order multiple scale solution carried out on the equations of motion expanded up to the fifth-order terms, along with the effects of nonlinear damping on the limit cycle amplitude for different linearly damped columns.

Singh, Yeong, Zhang, Rapti and Sri Namachchivaya consider an 11-dimensional stochastic model, describing the dynamics of a two-degree-of-freedom nonlinear airfoil in a turbulent flow, close to the flutter condition. Stochastic dimensional reduction for nonwhite noise excitations is applied. This leads to a onedimensional stochastic differential equation, which captures the essential long-term dynamics. Stability is studied by calculating analytically the top Lyapunov exponent, which is compared with that obtained by numerical integration of the original system.

Mi and Gottlieb derive a nonlinear initial boundary value problem for a planar multi-tethered spherical aerostat system via the extended Hamilton's principle with geometric constraints, along with a single tether limiting case. The reduced-order modal dynamical systems for both single and multi-tether configurations are numerically investigated under harmonic incident flow excitation with negligible lift, revealing superharmonic, period-doubled, quasiperiodic and chaoticlike solutions close to a $3: 1$ internal resonance and to certain combination resonances.

Aiming to avoid multistability of systems for safety and reliability, Brzeski, Lazarek, Kapitaniak, Kurths and Perlikowski propose an algorithm to estimate the probability of reaching an expected solution in given ranges of initial conditions and parameters. Usefulness and strength of a basin stability approach in predicting the system behavior and selecting the parameters ranges where it behaves in a presumed way are highlighted for a tuned mass absorber, a piecewise smooth oscillator, and a multi-degree of freedom system, with relatively small computational effort.

De Leo, Simoneschi, Fabrizio and Di Egidio study the motion of a non-symmetric rigid block, equipped 
with an added pendulum, designed as a tuned-massdamper against base excitation. The equations of motion account for rocking, uplift and impact. A parametric analysis is performed via numerical integrations, by varying the eccentricity of the center of mass, the frequency and the amplitude of the excitation, as well the characteristics of the mass damper. It is shown that a proper design of the pendulum increases the amplitude of the excitation causing overturning.

Steindl addresses the problem of optimal deployment of a tethered satellite from a space ship. A lumped mass model of the tether is adopted, describing lateral oscillations of the string. The Pontryagin's maximum principle is applied to compute a timeoptimal deployment from a trivial downhanging configuration. It is shown that several bifurcations of different type occur, entailing discontinuous variations of the optimal solution candidates. Modelling and numerical difficulties offered by the problem are thoroughly discussed.

Adamiec-Wójcika, Awrejcewicz, Drąga and Wojciecha solve the nonlinear dynamics of a catenary riser undergoing heave excitation via the rigid finite element method, comparing with outcomes from finite difference or finite element methods. Its numerical effectiveness allows applications in dynamic optimization problems such as compensating the horizontal vibrations of a vessel/platform by vertical displacements of the upper end of the riser, thus ensuring that the maximum bending moment at a given point does not exceed its static counterpart.

Davini, Micheletti and Podio-Guidugli numerically study the impulsive dynamics of chains made of T3 tensegrity modules, focusing on the kinetic extensiontwist coupling which differentiates T3 chains from Fermi-Pasta-Ulam (FPU) lattices. Non-FPU chains are shown to support solitary traveling waves, as FPU ones. Yet, differences in propagation speed and behavior after reflections exist. The effects of boundary conditions and of inhomogeneity in the chain, due to the presence of both left- and right-handed modules, are addressed. The role of prestrain is also investigated.

\subsection{Identification and monitoring of structures}

Seven papers dealing with modal analysis, identification, and/or damage characterization of real structures, with also meaningful experimental contents, are a well-deserved tribute to Francesco's research outcomes [14-18] in this more applicationoriented area.

Bedon, Dilena and Morassi present the dynamical characterization of a cable-stayed bridge based on ambient vibration testing and operational modal analysis. Structural identification is carried out through a manual tuning procedure based on finite element models of increasing accuracy, which allows to improve the description of boundary conditions and mechanical interaction between the bridge components. Results from local dynamic testing allow to estimate the axial force in the cables and to assess the integrity of the suspending system of the bridge.

Piana, Lofrano, Carpinteri, Paolone and Ruta investigate the use of piezoelectric devices in the experimental modal analysis of structures. The setup consists of piezoelectric disk buzzers, usually employed in stringed musical instruments. The voltage signal generated by the piezoelectric, proportional to the specimen deformation, measures the average strain over the contact area. Laboratory tests are performed on metallic beam and arch, whose natural frequencies and modes are identified. Experimental and theoretical results are compared, showing high accuracy.

Gattulli, Lepidi, Potenza and Di Sabatino present a parametric model to describe the linearized free dynamics and the modal interactions of a cable-beam system, characterized by frequency crossing and veering phenomena between global modes, dominated by the beam dynamics, and local modes, dominated by cable vibrations. The theoretical findings are used to give a tentative interpretation of some unexpected cable-wall interactions observed during dynamic testing and monitoring of a historic church, heavily damaged by the 2009 L'Aquila earthquake.

Foti and Perotti propose a sound approach to the analytical estimate of the cable internal damping, starting from a recent mechanical model of a strand from which a simplified model of the hysteretic bending behavior of stranded cables is derived. The novel proposal is compared, with remarkably good results, with empirical literature equations and available experimental data for a wide range of strands and parameters that characterize practically most of the configurations commonly used in overhead electrical lines. 
Capecchi, Ciambella, Pau and Vestroni propose a damage identification technique in which not only frequencies, but also modal shapes are used. It is proved that a penalty term in the objective function, which weighs the difference between natural frequencies and modal displacements in the damaged and undamaged states, improves the local convexity of the objective function. This entails reduction of the illconditioning of the inverse problem, enhancement of convergence and a more accurate estimation of damage. The algorithm is validated against experimental tests.

To overcome the low accuracy of identified modal models in vibration based damage localization, Bernal and Kunwar present a localization scheme that avoids identification by operating with Fourier transforms of the output signals plus a model of the reference state. The approach differs from a model updating framework in that only the damage distribution enters the formulation. The method is also experimentally tested, and is well suited when the effective loading is difficult to measure but invariance of the spatial distribution is readily satisfied.

Gentile, Guidobaldi and Saisi present a vibrationbased structural health monitoring methodology suitable to historic masonry towers, by exemplifying its application in the continuous dynamic monitoring of a tall tower. The approach is based on the installation of low-cost monitoring systems and on the combined use of automated operational modal analysis, regression models to mitigate the environmental effects on identified natural frequencies, and multivariate statistical tools to detect the occurrence of abnormal structural changes.

\subsection{Solid mechanics}

The last two papers deal with problems in solid mechanics.

Addessi and Sacco present a multiscale approach for the in-plane analysis of masonry walls, by considering a standard 2D kinematics at the macroscale and an enriched plane state model at the microscale. A homogenization procedure is applied by deriving the masonry overall mechanical response on the basis of the study of a unit cell. A damagefriction model is considered for the mortar and a damage model for the brick. Localization problems of strains and damage due to material softening responses are overcome by using nonlocal integral formulations.

Marmo, Toraldo and Rosati analyze the displacement field, strain and stress of a transversely isotropic elastic half-space, subject to linearly distributed vertical pressures. The surface integrals extended to polygonal regions are first transformed into boundary integrals and then reduced to algebraic sums at corners. Attention is payed to singularities. The algorithm holds for real, complex conjugate and coincident eigenvalues of the characteristic equation. Design charts for relevant quantities are provided. The influence of transversal isotropy against isotropy is investigated.

We conclude by sincerely thanking all of Francesco's colleagues and friends who agreed to take part in this initiative to honor his memory, and by gratefully acknowledging the availability of the Editor-in-Chief of Meccanica, Professor Luigi Gambarotta, to host their contributions in this journal.

\section{References}

1. Benedettini F, Rega G, Vestroni F (1986) Modal coupling in the free nonplanar finite motion of an elastic cable. Meccanica 21:38-46

2. Benedettini F, Rega G (1987) Nonlinear dynamics of an elastic cable under planar excitation. Int J Non-linear Mech 22:497-509

3. Benedettini F, Rega G, Alaggio R (1995) Nonlinear oscillations of a four-degree-of-freedom model of a suspended cable under multiple internal resonance conditions. J Sound Vib 182:775-798

4. Benedettini F, Rega G, Salvatori A (1992) Prediction of bifurcation and chaos for an asymmetric elastic oscillator. Chaos Solit Fractals 2:303-321

5. Rega G, Salvatori A, Benedettini F (1995) Numerical and geometrical analysis of bifurcation and chaos for an asymmetric elastic nonlinear oscillator. Nonlinear Dyn 7:249-272

6. Benedettini F, Moon FC (1995) Experimental dynamics of a hanging cable carrying two concentrated masses. Int $\mathrm{J}$ Bifurc Chaos 5:145-157

7. Rega G, Alaggio R, Benedettini F (1997) Experimental investigation of the nonlinear response of a hanging cable. Part I: local analysis. Nonlinear Dyn 14:89-117

8. Zulli D, Alaggio R, Benedettini F (2009) Nonlinear dynamics of a curved beam. Part II: numerical analysis and experiments. Int J Non-linear Mech 44:630-643

9. Benedettini F, Alaggio R, Zulli D (2012) Nonlinear coupling and instability in the forced dynamics of a non-shallow arch: theory and experiments. Nonlinear Dyn 68:505-517 
10. Benedettini F (1996) An experimental time series analysis approach in the classification of non periodic motions in nonlinear structural dynamics. In: Augusti G et al (eds) Structural dynamics, vol 1. Balkema, Rotterdam, pp 415421. ISBN: 9054108134

11. Benedettini F, Rega G (1997) Experimental investigation of the nonlinear response of a hanging cable. Part II: global analysis. Nonlinear Dyn 14:119-138

12. Benedettini F, Alaggio R (2003) The role of experimental tests in the formulation of reduced order analytical models in the planar dynamics of circular arches. In: Luongo A (ed) Recent research in structural dynamics. Research Signpost, Trivandrum, pp 285-297. ISBN:81-7736-186-4

13. Benedettini F, Capecchi D, Vestroni F (1995) Identification of hysteretic oscillators under earthquake loading by nonparametric models. J Eng Mech 121:606-612

14. Parloo E, Cauberghe B, Benedettini F, Alaggio R, Guillame $P$ (2005) Sensitivity-based operational mode shape normalization: application to a bridge. Mech Syst Signal Process 19:43-55

15. Benedettini F, Gentile C (2011) Operational modal testing and FE model tuning of a cable-stayed bridge. Eng Struct 33:2063-2073

16. Benedettini F, Morassi A, Vestroni F (2012) Structural assessment of bridges and health monitoring programs based on dynamical tests. In: Biondini F, Frangopol D (eds) Bridge maintenance, safety, management, resilience and sustainability: Proceedings of the Sixth International IABMAS Conference, Stresa, Lake Maggiore, Italy, 8-12 July 2012. CRC Press, pp 2792-2799. ISBN:9780415621243

17. Benedettini F, Dilena M, Morassi A (2015) Vibration analysis and structural identification of a curved multi-span viaduct. Mech Syst Signal Process 54:84-107

18. Benedettini F, De Sortis A, Milana G (2016) In field data to correctly characterize the seismic response of buildings and bridges. Bull Earth Eng. doi:10.1007/s10518-016-9917-4 